Review Article

\title{
Bayesian Network Meta-Analysis of the Effectiveness of Various Interventions for Nontraumatic Osteonecrosis of the Femoral Head
}

\author{
Ji Wang $\mathbb{D},{ }^{1}$ Jing Wang, ${ }^{2}$ Kai Zhang, ${ }^{3}$ Yanfang Wang $\mathbb{D},{ }^{4}$ and Xuanwen Bao $\mathbb{D}{ }^{2}$ \\ ${ }^{1}$ Department of Orthopedics, No. 210 Hospital of the PLA, Dalian, Liaoning Province 116000, China \\ ${ }^{2}$ Technical University Munich (TUM), 80333 Munich, Germany \\ ${ }^{3}$ Department of Cardiology, Sir Run Run Shaw Hospital, Zhejiang University School of Medicine, Hangzhou 310016, China \\ ${ }^{4}$ Ludwig-Maximilians-Universität München (LMU), 80539 Munich, Germany
}

Correspondence should be addressed to Yanfang Wang; yangfang.wang@cup.uni-muenchen.de and Xuanwen Bao; xuanwen.bao@helmholtz-muenchen.de

Received 29 April 2018; Accepted 26 July 2018; Published 6 August 2018

Academic Editor: Lei Yao

Copyright (C) 2018 Ji Wang et al. This is an open access article distributed under the Creative Commons Attribution License, which permits unrestricted use, distribution, and reproduction in any medium, provided the original work is properly cited.

Objective. To assess the effectiveness of various therapeutic hip preservation strategies on patients with nontraumatic osteonecrosis of the femoral head (ONFH). Design. This is a systematic review of previous literature and in-depth Bayesian network meta-analysis of randomized controlled trials (RCTs) to compare the clinical effect of various operation methods and one physical intervention (extracorporeal shockwave). Data Sources. Electronic literature, for studies published up to December 2017, was collected from PubMed, Medline, and the Cochrane Library. Study Selection. We selected RCTs on patients with ONFH. Treatment methods included extracorporeal shockwave (ESW), core decompression (CD), multiple drilling decompression (DD), vascularized fibular grafting (VFG), free-vascularized fibular grafting (FVFG), inverted femoral head grafting (IFHG), vascular iliac pedicle bone grafting (VIPBG), osteotomy, and tantalum implantation (TI). Outcome. The primary outcome was Harris score; the secondary outcome was Harris hip score (HHS), including total hip arthroplasty requirement (THA) and progression to collapse. Results. A total of 14 randomized controlled trials were investigated. ESW had the highest improvement on Harris score (probability best $52 \%$ ), followed by VFG (probability was 38\%). In the meanwhile, VFG also proved to be superior in reducing the failure rates of treatment (probability lowest 59\%), followed by ESW (probability lowest 24\%). In femoral necrosis stage-II, VFG achieved the highest probability in preventing treatment failures (52\%) and showed better performance in reducing treatment failure rates than CD. Conclusion. ESW therapy (ESWT) is the most effective intervention to improve HHS, and VFG shows superior effect on reducing treatment failure rates.

\section{Introduction}

Osteonecrosis of the femoral head (ONFH) is a debilitating disorder with a considerably high incidence in individuals aged between the third and fifth decades of life. In the United States, ONFH is reported to affect 20,000 patients each year, and it is estimated that more than $10 \%$ of ONFN patients eventually required total hip arthroplasties (THA) annually [1]. ONFH is histologically characterized by insufficient supply of blood, death of osteocytes, and bone marrow cells, as well as progressive structure damage of involved bones, which typically follows a progressive course leading to femoral head collapse and hip joint destruction. Therefore, how to retard the progression of ONFH is always a research hotspot.

The principle of ONFH treatment includes the termination of pathologic progression and the restoring of weightbearing capacity. Nowadays, the treatment and management for ONFH consists of conservative and surgical approaches. In a large number of studies, the clinical effect of ESWT which was assessed from a comprehensive perspective showed better effect than other conservative therapeutic methods [2, 3]. Besides, some RCT researches were designed to compare different surgical interventions for $\mathrm{ONFH}$, including $\mathrm{CD}$, DD, bone transplantation (VFG, FVFG, VIPBG, and IFHG), 
Osteotomy, and TI. These surgical interventions have been applied to avoid the most invasive intervention: total hip arthroplasty (THA). Core decompression, which is strictly limited to the treatment for early stages of ONFH before femoral head collapse [4], is now one of the most frequently used interventions and offers effective outcomes. However, some studies manifested that vascularized bone grafting (e.g., VFG, VIPBG) was superior to other treatments in clinical outcomes, radiographic findings, and durability of treatments $[5,6]$, though the operation method of grafting process and the location of the grafts may have a significant influence on the therapeutic effect. Above all, the optimal intervention to preserve the femoral head remains controversial. In this study, a Bayesian network meta-analysis method was used for the quantitative comparison of different surgical interventions and ESWT to determine the optimal treatment for ONFH.

\section{Method}

2.1. Data Sources and Searches. We searched literature published between 1980 and 2017 in three electronic databases (Medline, PubMed, and the Cochrane Library) for randomized clinical trials investigating operation methods for ONFH, with Medical Subject Headings (MeSH) and text words.

For the purpose of Bayesian network meta-analysis, the search extracted trials comparing ESW, VFG, FVFG, CD, DD, IFHG, TI, osteotomy, and VIPBG. The trials involving cellular therapy or biomaterials were excluded.

2.2. Study Selection. We included randomized, parallel group design clinical trials comparing the effects of chosen operation methods and one physical intervention (ESW).

Inclusion criteria for studies were as follows: (1) the study should be randomized, parallel group design clinical trial; (2) the study should focus on ONFH; (3) the included studies should report at least one of the three outcomes: the improvement of Harris score and the frequency that hips required THA or underwent collapse of the femoral head after intervention.

Exclusion criteria were as follows: (1) the study was not a RCT design; (2) the study included non-ONFH patients; (3) the study combined cell-therapy effect with surgical options (e.g., VFG plus mesenchymal stem cell implantation). Eligible studies should be published as full-length articles or letters in peer-reviewed journals.

2.3. Data Extraction and Quality Assessment. Two investigators independently extracted the following information: study design; name of the first author, publication year; sample size, patients' characteristics; interventions; comparisons; outcomes (postoperative Harris score and treatment failure rate) and follow-up. Two investigators independently evaluated the methodological quality of eligible RCTs by the Cochrane Collaboration's tool [7] for assessing risk of bias on the basis of (1) random sequence generation, (2) allocation concealment, (3) blinding of participants and personnel, (4) blinding of outcome assessment, (5) incomplete outcome data, (6) selective reporting, and (7) other sources of bias.

2.4. Outcome Assessment. HHS and treatment failure rates were regarded as the outcome of the included RCTs. HHS includes four subscales: (1) range of motion, (2) joint activity, (3) pain, and (4) absence of deformity. A higher score indicates a better treatment outcome. Treatment failure rates included the frequency of THA requirement and the collapse of the femoral head after intervention. If both events were reported in RCTs, we chose the outcomes with greater number.

2.5. Statistical Methods. Quantitative data of each eligible study were first extracted in a spreadsheet. We analyzed two treatment outcomes separately (HHS and treatment failure rates). STATA (version 13.0, StataCorp LLC,TX, USA) and the GeMTC R package (version 3.2.3, R Development Core Team/R Foundation for Statistical Computing, Vienna, Austria) were used to perform network meta-analysis with Bayesian hierarchical random-effects model [8]. The random-effects model allowed the heterogeneity among trials on the assumption that treatment effects originated from a normal distribution. Markov Chain Monte Carlo (MCMC) simulation method was used to calculate the posterior distributions of the nodes in Bayesian network frame. Noninformative priors with vague normal (mean 0 , variance 10 000) and uniform (0-2) prior distributions for outcomes such as HHS and treatment failure rates were performed [8]. Sensitivity analysis was performed to examine the effects of different operation methods. We generated 100000 simulations for each initial value, and the first 50000 simulations were discarded. Convergence was assessed by Brooks-Gelman-Rubin method. The median of the posterior was the point estimate and the corresponding 95\% credible intervals using the 2.5 th and 97.5 th percentiles of the posterior distribution were reported as $95 \%$ credibility intervals [9]. Inconsistency was evaluated in a loop which connected three treatments [10]. We used node splitting method to evaluate the inconsistency of network model [11, 12]. A particular comparison based on direct and indirect evidence was performed, in which Bayesian $\mathrm{P}>0.05$ was regarded as insignificant [12]. The probability of each treatment being the best was calculated to provide a more comprehensible measure of treatment efficacy on the basis of its posterior probability. The plots of ranking probabilities (rankograms) illustrated the order of treatments for each outcome [13].

Potential publication bias was assessed by visual inspection of comparison-adjusted funnel plot [14-16]. The Cochran's $\mathrm{Q}\left(\mathrm{X}^{2}\right)$ and the $\mathrm{I}^{2}$ statistical test were calculated to test for statistical heterogeneity [17].

\section{Results}

3.1. Characteristics and Quality Evaluation of Included Studies. We retrieved 1209 related publications in total, among which 292 duplications, 140 letters or reviews, and 163 non-English publications were immediately eliminated. 584 publications were excluded by screening their titles and abstracts. The 

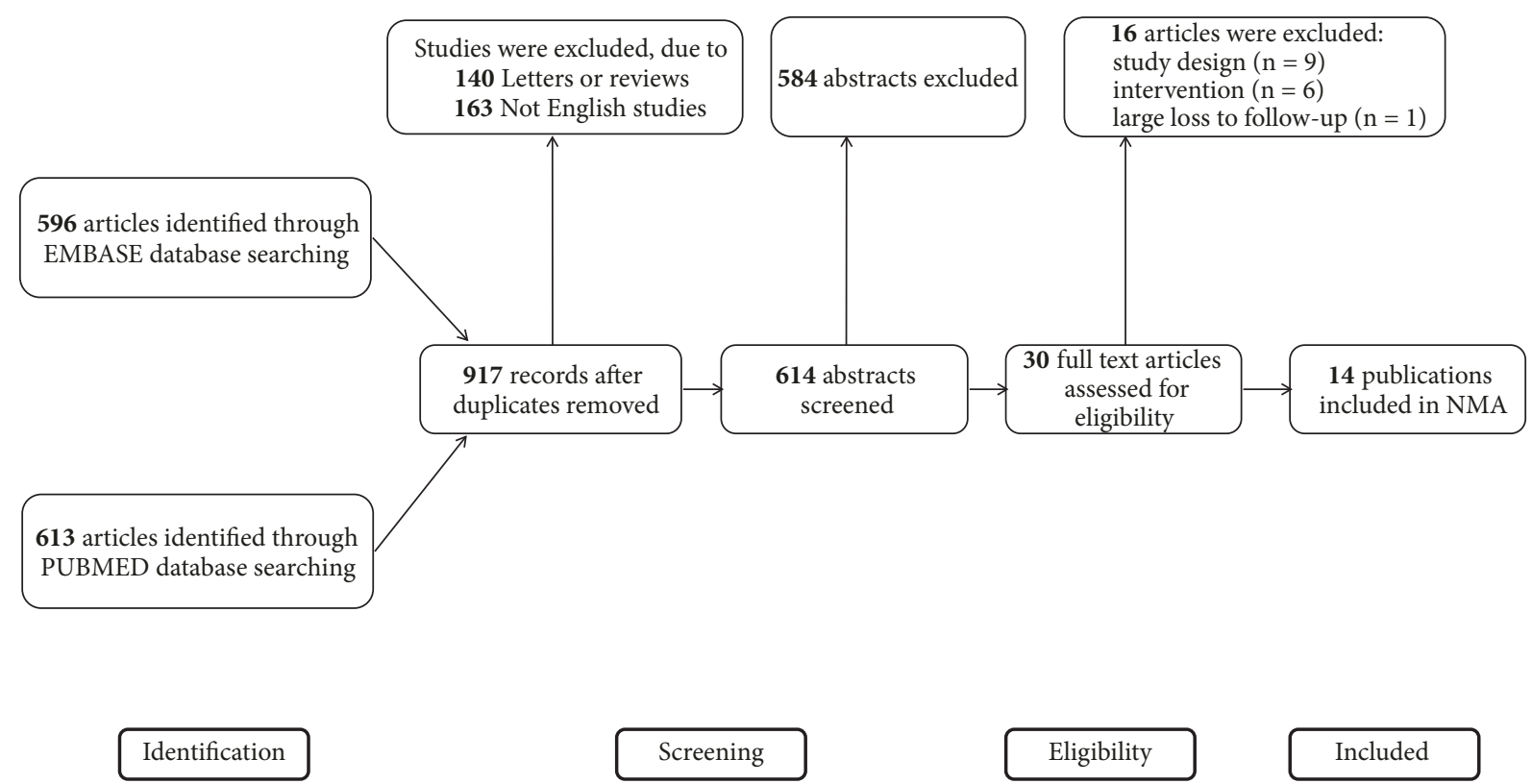

FIGURE 1: PRISMA flowchart illustrating the selection of studies included in this study.

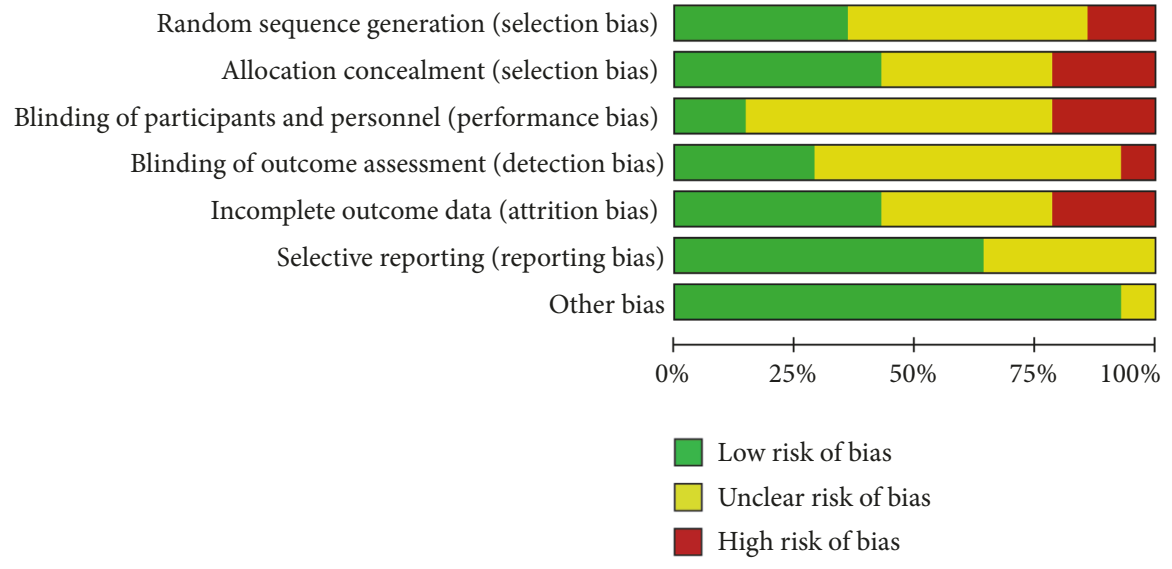

FIGURE 2: Risk of bias graph for each included study.

entire process of literature searching and screening for NMA was illustrated in Figure 1. With the full-text assessment, 14 RCTs with a number of 1298 patients were enrolled into our analysis. Main study endpoints included HHS, the treatment failure rates of femoral necrosis in total stage and stage-II. Baseline characteristics of the 14 studies were presented in Table 1. All of the included studies were RCTs; the overall quality of included studies was embodied in Figure 2.

3.2. Network Meta-Analysis of HHS. 10 two-arm RCT studies reported the HHS, recruiting 538 patients (Figure 3(a)). These studies were incorporated into the present network Bayesian model. The mean duration of follow-up was 22 months. VFG was the most frequently studied intervention, and the comparisons between VFG and FVFG were more than those in other studies. In the direct pairwise comparisons, enhancement for VFG was statistically significant as compared with FVFG (MD=16, 95\% CrI [2.3, 29]). Other pairwise comparisons did not show significant outcome.

In random-effects relative forest plots, enhancement was detected with statistical significance for ESW versus DD (MD $=29,95 \%$ CrI $[1.6,57])$, VFG versus DD, FVFG, and IFHG $(\mathrm{MD}=28,95 \% \operatorname{CrI}[6.8,49] ; \mathrm{MD}=16,95 \% \operatorname{CrI}[2.3,29] ; \mathrm{MD}$ $=39,95 \%$ CrI $[0.56,78])$; there was no significant effect on improving HHS among the other interventions (Figure 4(a)).

In rankograms (Figure 6(a)), ESW was estimated to have a $55.4 \%$ chance of being the best intervention for the treatments of femoral necrosis, followed by VFG (38.6\%). From the perspective of improving HHS, our study revealed that ESW therapy and VFG technique were the best two options for hip preservation treatments in terms of improving HHS.

3.3. Network Meta-Analysis of the Treatment Failure Rates in Femoral Necrosis Total Stage. In our study, treatment failure 


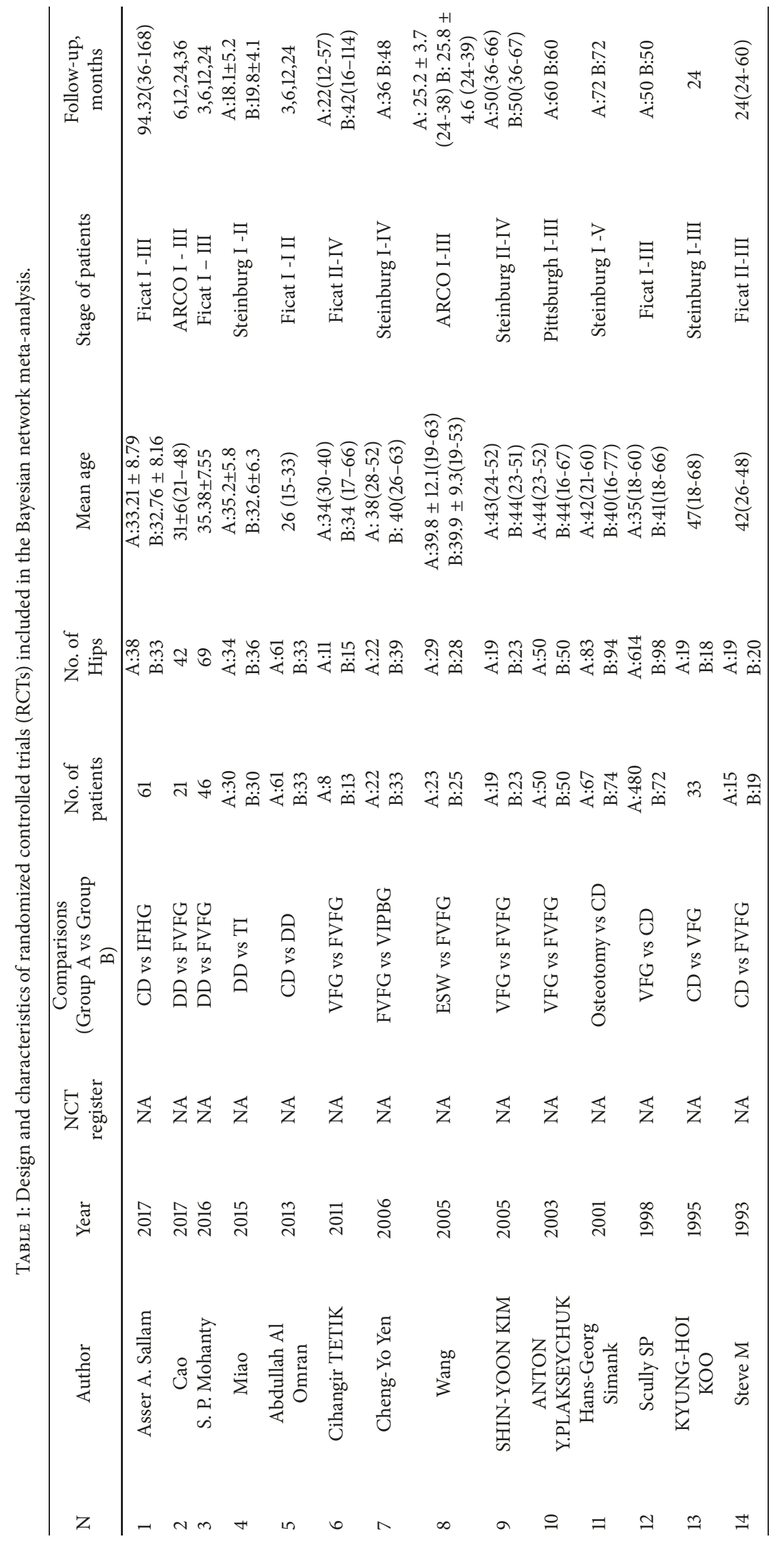




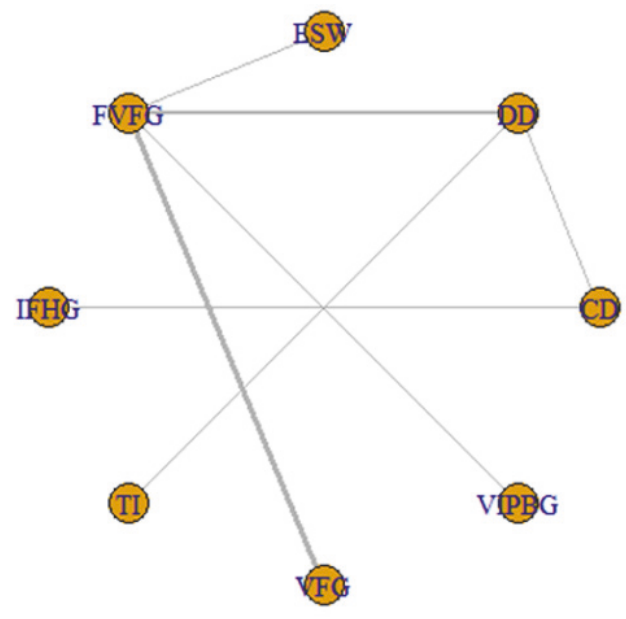

(a) Harris hip score

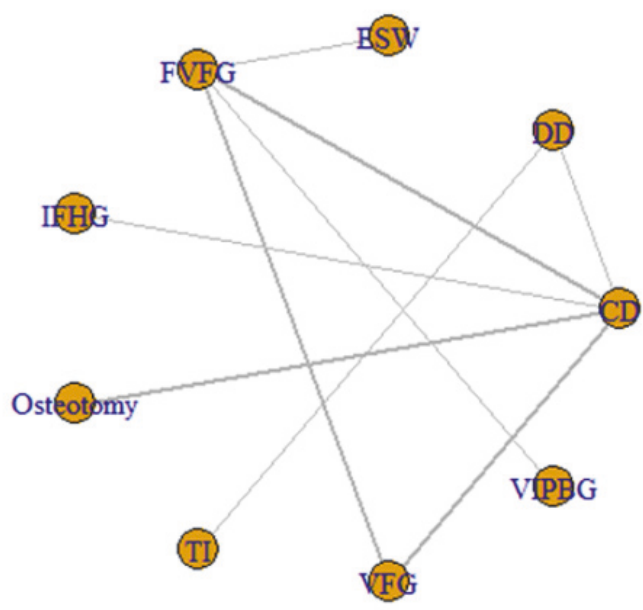

(b) Treatment failure rates

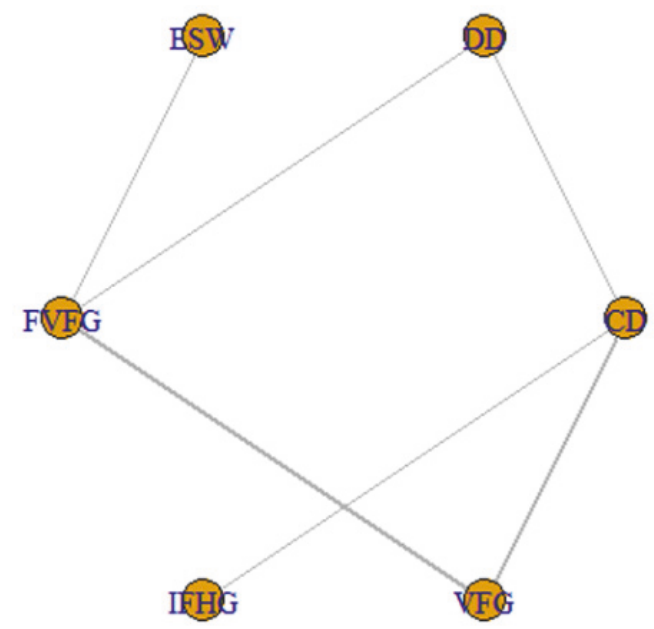

(c) Treatment failure rates in stage-II

FIGURE 3: Network comparisons for (a) HHS, (b) the treatment failure rates (Collapse \& THA progression), and (c) the treatment failure rates (Collapse \& THA progression) in stage-II included in the analyses. THA: total hip arthroplasty; CD: core decompression; ESWT: extracorporeal shock wave; DD: multiple drilling decompression; VFG: vascularized fibular grafting; FVFG: free-vascularized fibular grafting; IFHG: inverted femoral head grafting (IFHG); VIPBG: vascular iliac pedicle bone grafting; TI: tantalum implantation.

outcomes included collapse and THA progression. There were 13 literature sources that described the treatment failure rates, recruiting 1277 patients (Figure 3(b)). In the direct pairwise comparison, the overall effect showed that VFG had a statistically lower treatment failure rate as compared to $\mathrm{CD}$ $(\mathrm{OR}=0.16,95 \% \mathrm{CrI}[0.048,0.39])$, while there was no significant difference among other interventions (Figure 4(b)).

In random-effects relative forest plots, VFG had a lower treatment failure rate $(\mathrm{OR}=0.16,95 \% \mathrm{CrI}[0.048,0.39]$; $\mathrm{OR}=$ $0.12,95 \%$ CrI $[0.042,0.37] ;$ OR $=0.18,95 \%$ CrI $[0.034,0.94])$ compared with CD, FVFG, and osteotomy for total stage (Figure 5(b)). There was no significant difference in treatment failure rates for other methods. Rankograms indicated that VFG had the lowest probability for the progression to THA and femoral collapse. Besides, ESW also showed a low probability for the progression to treatment failures (Figure 6(b)).

3.4. Network Meta-Analysis of the Treatment Failure Rates in Femoral Necrosis Stage-II. A total of 13 studies described the treatment failure rates in femoral necrosis stage-II (Figure 3(c)). The overall effect showed that only VFG had a statistically lower failure rate as compared with $\mathrm{CD}(\mathrm{OR}=0.13,95 \%$ CrI $[0.0036,1.0]$ ) in the direct pairwise NMA (Figure 4(c)). Moreover, we found that VFG tended to show lower failure rates than FVFG in random-effects relative forest plots (OR $=0.090,95 \%$ CrI $[0.0053,1.1]$ ) (Figure 5(c)). Rankograms indicated that VFG was the optimal intervention in the prevention of treatment failures (Figure 6(c)). 


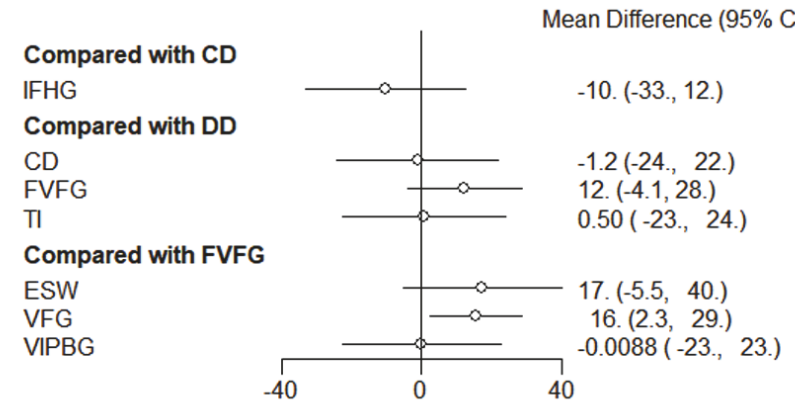

(a) Harris hip scores

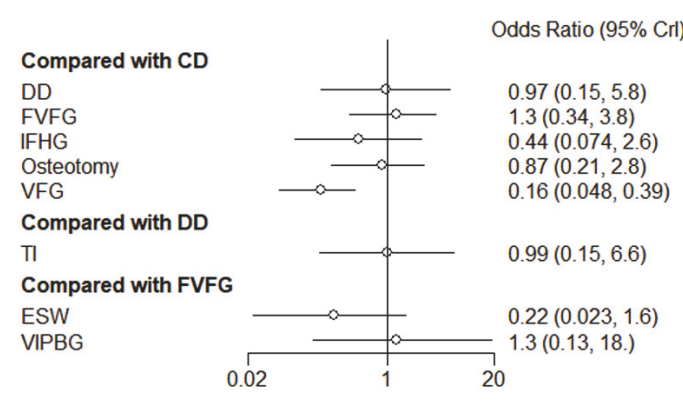

(b) Treatment failure rate

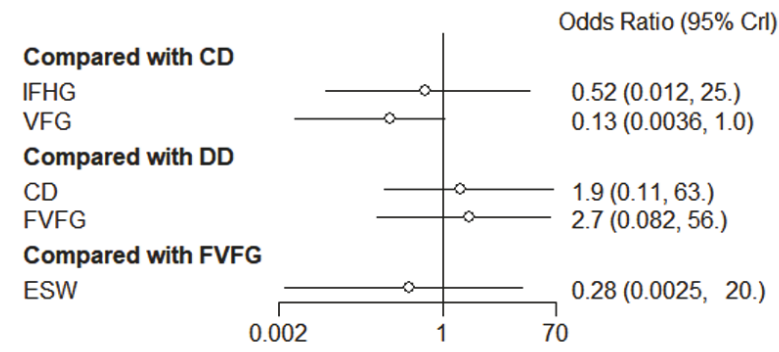

(c) Treatment failure rates in femoral necrosis stage-II

FIGURE 4: Forest plots of direct comparison of (a) HHS and (b) the treatment failure rates included in the network meta-analysis in a Bayesian framework. CD: core decompression; ESWT: extracorporeal shock wave; DD: multiple drilling decompression; VFG: vascularized fibular grafting; FVFG: free-vascularized fibular grafting; IFHG: inverted femoral head grafting (IFHG); VIPBG: vascular iliac pedicle bone grafting; TI: tantalum implantation.

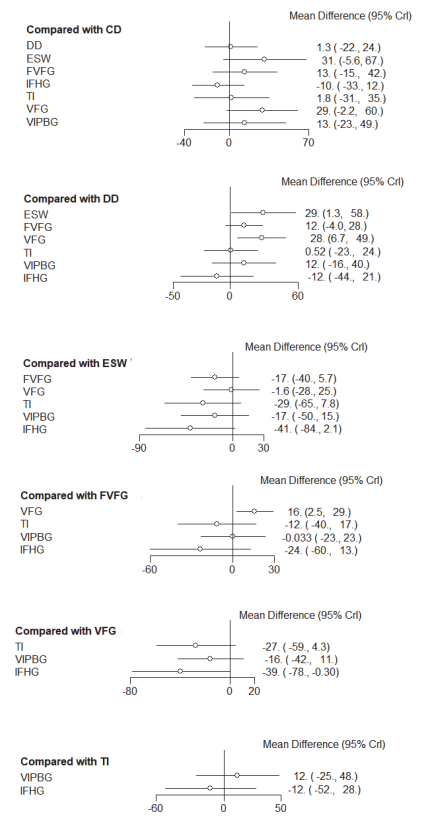

(a) Harris hip score
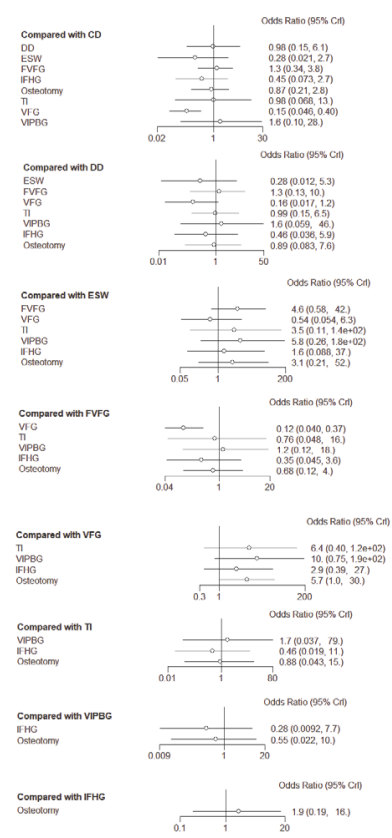

(b) Treatment failure rates
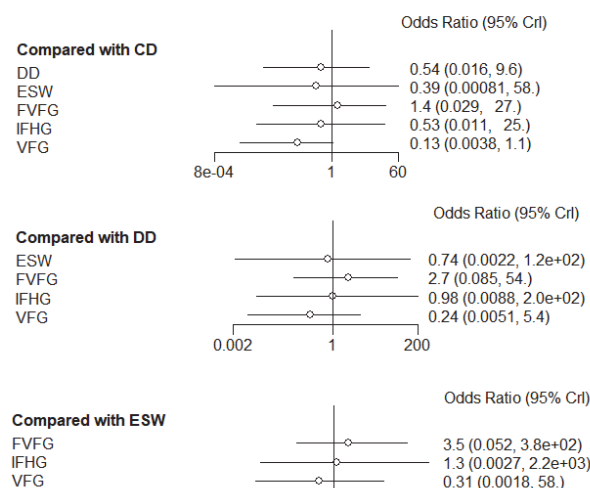

FVFG
IFHG
VFG
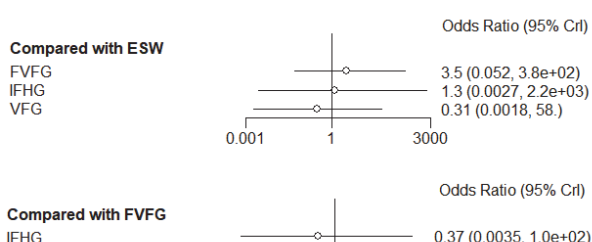

IFHG
VFG

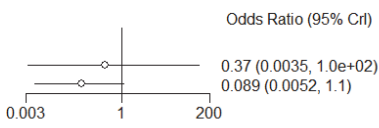

Compared with VFG

IFHG

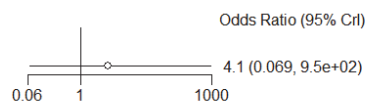

(c) Treatment failure rates in stage-II

FIGURE 5: Relative forest plots of each pairwise comparison of (a) HHS, (b) the treatment failure rates, and (c) the treatment failure rates in stage-II included in the network meta-analysis in a Bayesian framework. THA: total hip arthroplasty; CD: core decompression; ESWT: extracorporeal shock wave; DD: multiple drilling decompression; VFG: vascularized fibular grafting; FVFG: free-vascularized fibular grafting; IFHG: inverted femoral head grafting (IFHG); VIPBG: vascular iliac pedicle bone grafting; TI: tantalum implantation. 


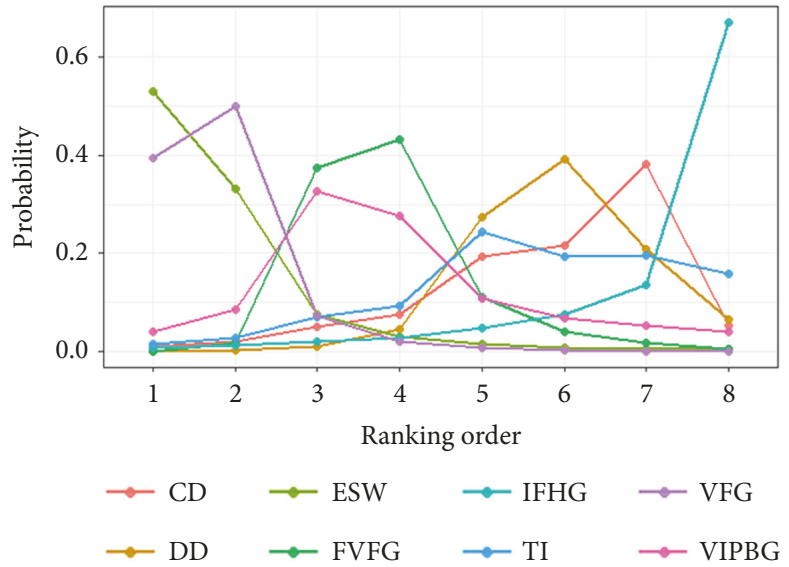

(a) Harris hip score

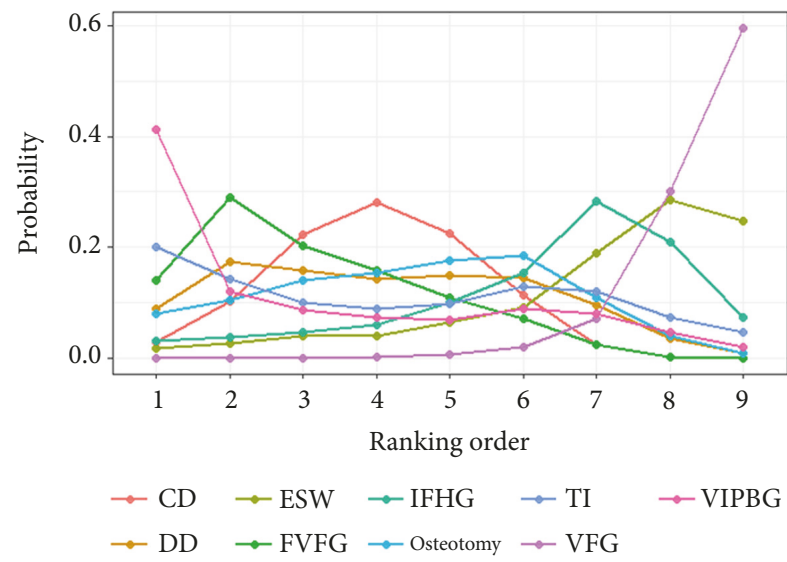

(b) Treatment failure rates

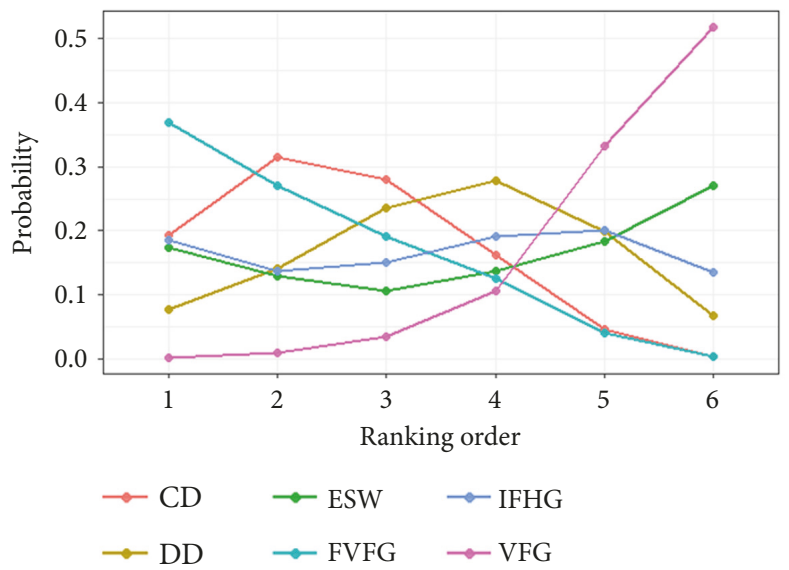

(c) Treatment failure rates in stage-II

FIGURE 6: Rank probability curves for (a) HHS, (b) the treatment failure rates, and (c) the treatment failure rates in stage-II. Distribution of probabilities for each treatment is ranked at different positions for each outcome. CD: core decompression; ESWT: extracorporeal shock wave; DD: multiple drilling decompression; VFG: vascularized fibular grafting; FVFG: free-vascularized fibular grafting; IFHG: inverted femoral head grafting (IFHG); VIPBG: vascular iliac pedicle bone grafting; TI: tantalum implantation.

3.5. Inconsistence Check. Statistical consistency between direct and indirect comparisons for HHS and treatment failure rates in total stage and stage-II was analyzed in Figure 7. The overall network was highly consistent, without significant differences between direct and indirect comparisons.

3.6. Funnel Plot and Publication Bias. The funnel plot of HHS and treatment failure rates were shown in Figures 8(a) and 8 (b), respectively. Symmetrical Scatters were observed in the funnel plot, manifesting the publication bias in the results of HHS, and treatment failure rates events in various studies were relatively low.

\section{Discussion}

How to postpone ONFH progression remains a challenge that needs to be fully understood. Although there are varieties of treatments for preserving the femoral head [18], the optimal one is still controversial. In the present Bayesian network meta-analysis study, we systematically analyzed the effects of several hip preservation treatments on nontraumatic
ONFH, including ESW, CD, DD, VFG, FVFG, IFHG, VIPBG, osteotomy, and TI.

$\mathrm{CD}$ is a standard technique widely used in patients with early-stage ONFH, which helps to decrease intraosseous pressure in the femoral head, reestablish vascular flow, and relieve pain [19]. There were many studies that investigated the efficacy of CD for the treatment of patients with earlystage ONFH. Nevertheless, the clinical success of CD has inconsistencies (from $47 \%$ to $83 \%$ ), mainly related to many different factors including causes and the stage of the lesion, skill of procedures, and duration of follow-up [20-23]. Based on our network results and rankings results, CD provided a moderate outcome in terms of improving HHS and preventing treatment failures as compared with other jointpreserving treatments.

Some articles indicated that the conventional core decompression might lead to further collapse of femur head due to the weakening of subchondral bone support [24, 25]. To overcome the limitations of this surgery, multiple drilling has been advocated. One research demonstrated that multiple drilling effectively reduced the pressure of femoral 


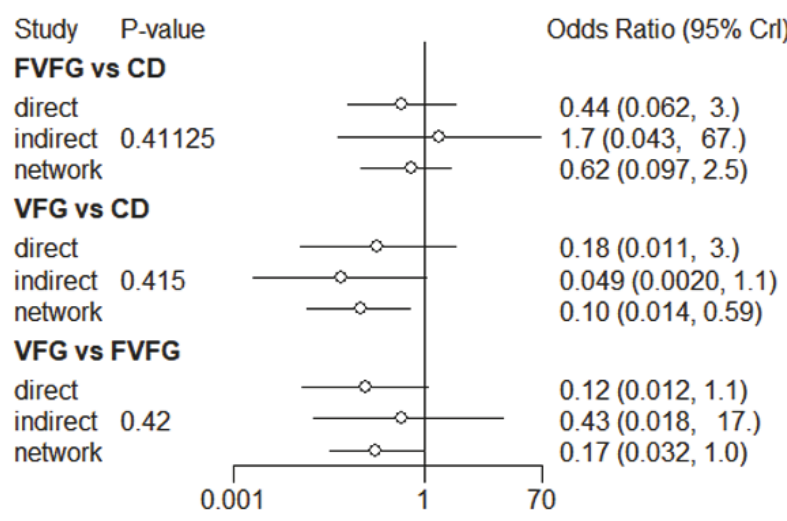

(a) Harris hip score

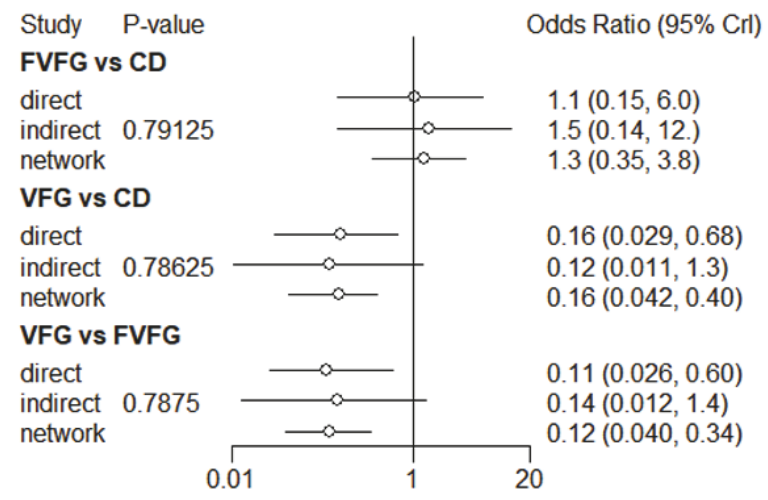

(b) Treatment failure rates

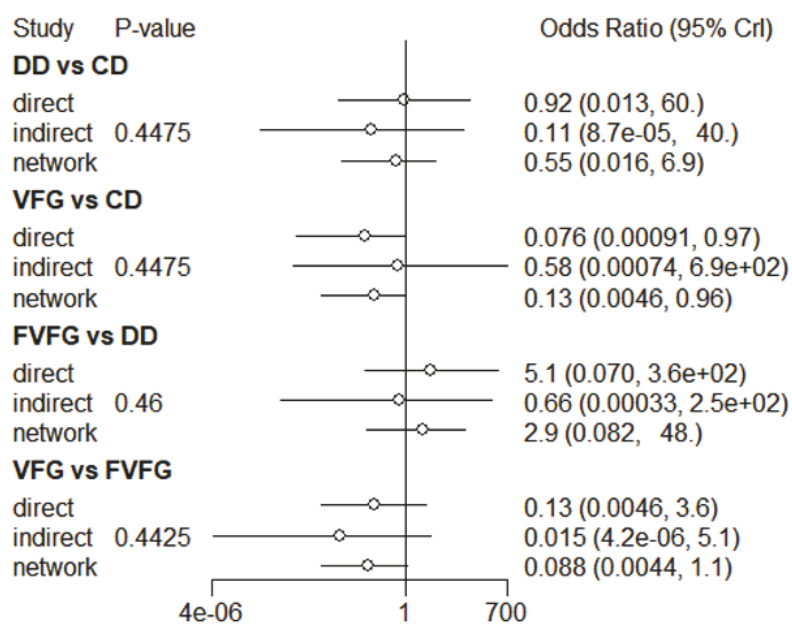

(c) Treatment failure rates in stage-II

FIGURE 7: Forest plot of network consistency analysis for comparisons by node splitting method. Numbers represent the posterior means and standard deviations (SDs) of the direct, indirect, and network estimates of the odds ratios (ORs) and rate ratios (RRs). CD: core decompression; ESWT: extracorporeal shock wave; DD: multiple drilling decompression; VFG: vascularized fibular grafting; FVFG: free-vascularized fibular grafting; IFHG: inverted femoral head grafting (IFHG); VIPBG: vascular iliac pedicle bone grafting; TI: tantalum implantation.

head and meanwhile maintained the supportive role of the subchondral bone to avoid fractures or collapse of the femoral head [26]. However, DD did not show significant better effect on both the improvement of HHS and the prevention of THA progression than CD in our network study, though DD had a higher probability to rank better from rankograms in these two aspects.

Based on our network results and rankograms, we recommended ESW as one of the optimal interventions due to its better efficacy and moderate safety in improving HHS. The mechanism of ESW in the treatment of ONFH has not been fully understood [2]. ESW was equipped to spread to necrotic femoral hips, and a pressure loss of $50 \%$ of shockwave was observed $[27,28]$. Some researches proposed that ESW could induce microfracture to accelerate bone healing and enhance pain threshold [29-31]. Furthermore, ESW promoted bone healing by enhancing osteogenesis and angiogenesis as well as bone remodeling of diseased hips
$[32,33]$. In this study, rankogram of ESW showed the highest probability (52\%) of being the best treatment in terms of HHS improvement. Increased range of motion and joint activity as well as reduction of pain might be due to the promotion of tissue remolding and the bluntness of pain sensation, which would help the improvement of HHS. For the prevention of collapse and THA progression, ESW also had a satisfactory performance both in all stage and stage-II in rankogram, indicating that ESW was one of the most optimal ways in inhibiting progression.

The use of vascularized fibular graft was initiated in an effort to arrest the progression of necrosis and to enhance angiogenesis. The success of this surgical method was in connection with multiple factors: (1) sufficient decompression, (2) mechanical support, and (3) augmentation with additional cancellous bone graft. VFG not only provides structural support, but also restores vascular supply to enhance lesion healing $[34,35]$. A report on the long-term 


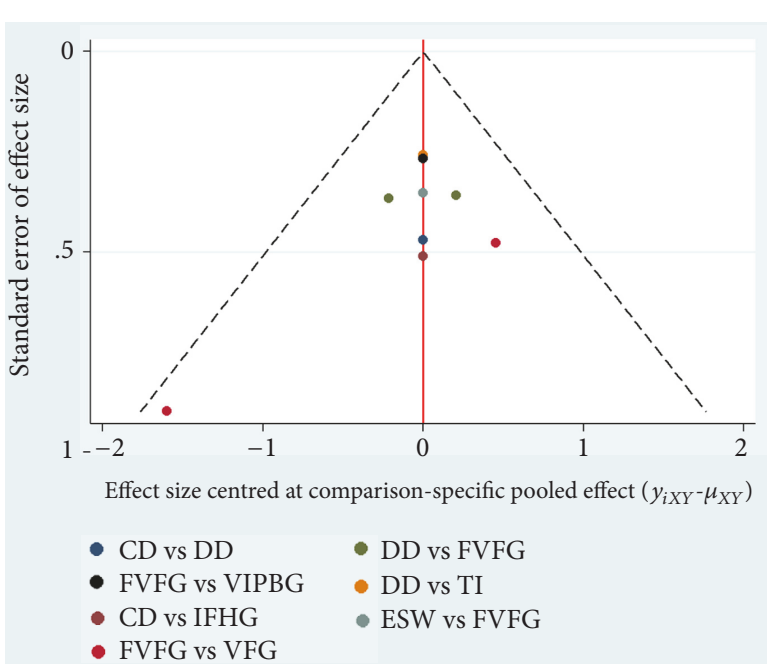

(a) Harris hip score

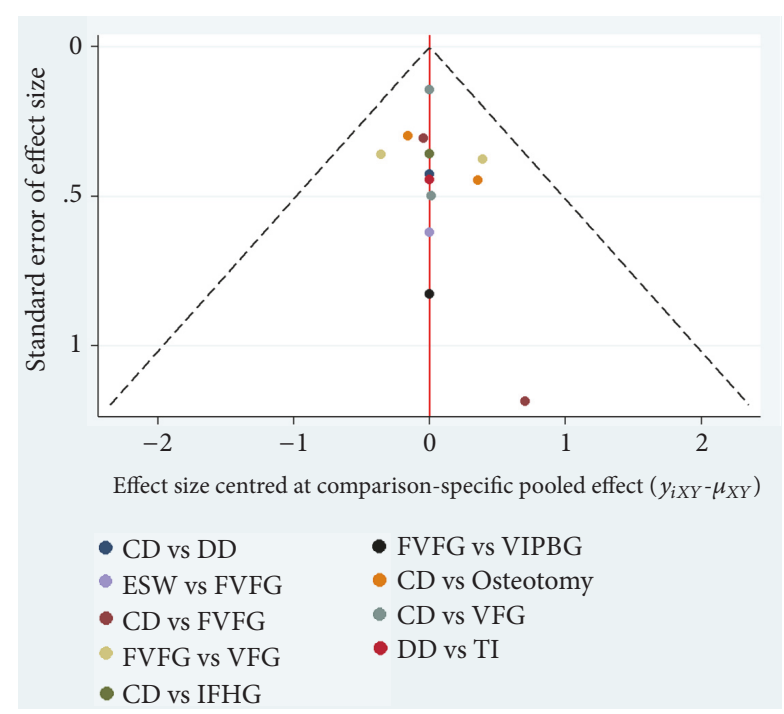

(b) Treatment failure rates

FIGURE 8: Comparison-adjusted funnel plot for assessment of (a) HHS and (b) the treatment failure rates. CD: core decompression; ESWT: extracorporeal shock wave; DD: multiple drilling decompression; VFG: vascularized fibular grafting; FVFG: free-vascularized fibular grafting; IFHG: inverted femoral head grafting (IFHG); VIPBG: vascular iliac pedicle bone grafting; TI: tantalum implantation.

results of VFG indicated that 49 of 65 hips (75\%) survived at a mean follow-up of fifteen years (range: 10.5-26.1 years) [36]. Moreover, some studies suggested that VFG was a better treatment option than $\mathrm{CD}$ and nonvascularized fibular grafting due to the less dome depression of the femoral head [37-39]. Our results showed VFG had significantly better effect in improving HHS than DD and FVFG. Besides, it also had a better outcome in terms of inhibiting collapse and THA progression than DD, FVFG, and osteotomy. Meanwhile, the rankogram showed VFG had the highest probability (59\%) of being the best treatment in preventing collapse and THA progression. In HHS improvement, VFG had 39\% probability of being the best treatment and 50\% probability of being the second best treatment. Hence, we got the conclusion that VFG would be one of the most optimal interventions.

TI has been used as reasonable mechanical substitute of a fibular graft following CD for ONFH treatment [40]. The tantalum implant aims to provide a structural scaffold for bone ingrowth by its osteoconductive capacity [41]. However, the effect of TI transplantation is still under debate. In one study, 16 of 58 hips (28\%) showed radiographic progression and 9 of 58 hips (16\%) converted to THA at a mean followup of 24 months [42]. Tanzer $M$ found the presence of bone ingrowth in thirteen (87\%) of the fifteen specimens by backscattered scanning electron microscopy. The mean extent of bone ingrowth was only $1.9 \%$ (range: $0 \%$ to $4.4 \%$ ). The retrieved implants were associated with limited bone ingrowth and insufficient mechanical support of subchondral bone [43]. Based on our study, TI did not have significantly different effect as compared with DD and CD, yet the effect of TI, DD, and CD was not as good as that of ESW and VFG.

Osteotomies aim to prevent femoral head collapse by transposing the osteonecrotic area from a weight-bearing to a non-weight-bearing area of the hip joint, thereby diverting mechanical stress from the lesion to healthy bone. An earlier study found that 28 hips (76\%) treated by intertrochanteric varus osteotomy had a good or excellent result at a mean follow-up of 11.5 years [44]. Another study indicated that 22 of 28 hips $(79 \%)$ treated by intertrochanteric rotational osteotomy had survived with a mean HHS of 85.8 at the final follow-up [45]. In recent years, the usage of osteotomy has been reduced due to limited indications for small lesions. Besides, osteotomies were associated with a higher rate of complications, such as nonunion or delayed union and loss of fixation and/or position, which also restricted the usage of this technique [46]. Moreover, this surgical technique was highly complicated, and it was difficult to convert the failed cases to THA. Based on the available data from our network analysis, we do not recommend this technology as the first choice until the publication of long-term follow-up results.

IFHG and VIG are two alternative interventions for ONFH. When IFHG was used, one cylindrical block of cancellous bone was harvested from the femoral head and then inverted and reinserted into the core. One study showed the improvement of HHS by IFHG compared with CD, although a proportion of patients experienced radiographic progression [47]. When VIPBG technique was used, vascularized iliac bone grafting was taken to replace the necrosis region [48]. In our study, the two interventions did not show significant difference in preventing collapse compared with other interventions. In terms of HHS, VFG showed slightly better outcome than IFHG. Thus, we would recommend VFG as a preferred choice for ONFH treatment compared with IFHG and VIG.

As far as we know, this was the first comprehensive network comparison of mainstream interventions for ONFH. To compare the efficacy of nine treatments for ONFH, we used a Bayesian network model to combine direct and 
indirect evidence on the effectiveness in 14 RCTs. From the analysis, we got the conclusion that VFG and ESW would be the optimal choices for the treatment of early ONFH in terms of improving HHS and preventing treatment failures. We also analyzed the patients by disease stage to get a better understanding of the efficacy of various interventions at different disease stages. As far as preventing treatment failures was concerned, VGF and ESW therapy also showed better outcomes in stage-II.

Nevertheless, this study had several limitations. First of all, only published studies were included in the present metaanalysis. Thus, publication bias may have occurred. Secondly, the heterogeneity existed in terms of risk factors, lesion sizes and stages, bilateral versus unilateral disease, surgical techniques, indications for THR, and follow-up time. Thirdly, detailed blind methods and allocation concealment were not described in some of the included RCTs, which could affect the validity for overall findings. Furthermore, due to the different classification measurements (radiographic evidence based on X-ray and MRI) applied in various reports, the reliability of the avascular necrosis status assessment was decreased.

\section{Conflicts of Interest}

The authors declare that there are no conflicts of interest regarding the publication of this paper.

\section{Authors' Contributions}

Ji Wang and Jing Wang contributed equally to this work.

\section{References}

[1] C. J. Lavernia, R. J. Sierra, and F. R. Grieco, "Osteonecrosis of the Femoral Head," Journal of the American Academy of Orthopaedic Surgeons, vol. 7, no. 4, pp. 250-261, 1999.

[2] E. M. Alves, A. T. Angrisani, and M. B. Santiago, "The use of extracorporeal shock waves in the treatment of osteonecrosis of the femoral head: a systematic review," Clinical Rheumatology, vol. 28, no. 11, pp. 1247-1251, 2009.

[3] J. Fessel, "There are many potential medical therapies for atraumatic osteonecrosis," Rheumatology, vol. 52, no. 2, pp. 235-241, 2013.

[4] J. r. Lieberman, D. J. Berry, M. A. Mont, R. K. Aaron, J. J. Callaghan, A. D. Rajadhyaksha et al., "Osteonecrosis of the hip: management in the 21st century," Instructional Course Lectures, vol. 52, pp. 337-355, 2003.

[5] A. Y. Plakseychuk, S.-Y. Kim, B.-C. Park, S. E. Varitimidis, H. E. Rubash, and D. G. Sotereanos, "Vascularized compared with nonvascularized fibular grafting for the treatment of osteonecrosis of the femoral head," The Journal of Bone \& Joint Surgery, vol. 85, no. 4, pp. 589-596, 2003.

[6] S. P. Scully, R. K. Aaron, and J. R. Urbaniak, "Survival analysis of hips treated with core decompression or vascularized fibular grafting because of avascular necrosis," Journal of Bone and Joint Surgery, vol. 80, no. 9, pp. 1270-1275, 1998.

[7] J. P. T. Higgins, D. G. Altman, P. C. Gøtzsche et al., "The Cochrane Collaboration's tool for assessing risk of bias in randomised trials," British Medical Journal, vol. 343, no. 7829, Article ID d5928, 2011.
[8] G. Lu and A. E. Ades, "Combination of direct and indirect evidence in mixed treatment comparisons," Statistics in Medicine, vol. 23, no. 20, pp. 3105-3124, 2004.

[9] S. Wandel, P. Jüni, B. Tendal et al., "Effects of glucosamine, chondroitin, or placebo in patients with osteoarthritis of hip or knee: Network meta-analysis," British Medical Journal, vol. 341, p. c4675, 2010.

[10] Y.-K. Tu, I. Needleman, L. Chambrone, H.-K. Lu, and C. M. Faggion Jr., "A bayesian network meta-analysis on comparisons of enamel matrix derivatives, guided tissue regeneration and their combination therapies," Journal of Clinical Periodontology, vol. 39, no. 3, pp. 303-314, 2012.

[11] S. Dias, N. J. Welton, D. M. Caldwell, and A. E. Ades, "Checking consistency in mixed treatment comparison meta-analysis," Statistics in Medicine, vol. 29, no. 7-8, pp. 932-944, 2010.

[12] G. Lu and A. E. Ades, "Assessing evidence inconsistency in mixed treatment comparisons," Journal of the American Statistical Association, vol. 101, no. 474, pp. 447-459, 2006.

[13] G. Salanti, A. E. Ades, and J. P. A. Ioannidis, "Graphical methods and numerical summaries for presenting results from multipletreatment meta-analysis: an overview and tutorial," Journal of Clinical Epidemiology, vol. 64, no. 2, pp. 163-171, 2011.

[14] M. Egger, G. D. Smith, M. Schneider, and C. Minder, "Bias in meta-analysis detected by a simple, graphical test," British Medical Journal, vol. 315, pp. 629-634, 1997.

[15] J. L. Peters, A. J. Sutton, D. R. Jones, K. R. Abrams, L. Rushton, and S. G. Moreno, "Assessing publication bias in meta-analyses in the presence of between-study heterogeneity," Journal of the Royal Statistical Society: Series A (Statistics in Society), vol. 173, no. 3, pp. 575-591, 2010.

[16] A. Chaimani, J. P. T. Higgins, D. Mavridis, P. Spyridonos, and G. Salanti, "Graphical tools for network meta-analysis in STATA," PLoS ONE, vol. 8, no. 10, Article ID e76654, 2013.

[17] J. P. Higgins and S. Green, "Cochrane handbook for systematic reviews of interventions," 2005.

[18] D. Van Der Jagt, L. Mokete, J. Pietrzak, C. G. Zalavras, and J. R. Lieberman, "Osteonecrosis of the femoral head: Evaluation and treatment," Journal of the American Academy of Orthopaedic Surgeons, vol. 23, no. 2, pp. 69-70, 2015.

[19] Y. C. Hong, H. M. Zhong, T. Lin, and J. B. Shi, "Comparison of core decompression and conservative treatment for avascular necrosis of femoral head at early stage: a meta-analysis," International Journal of Clinical and Experimental Medicine, vol. 8, no. 4, pp. 5207-5216, 2015.

[20] P. J. Brown, S. Mannava, T. M. Seyler, J. F. Plate, C. Van Sikes, and J. D. Stitzel, "Multiple small diameter drillings increase femoral neck stability compared with single large diameter femoral head core decompression technique for avascular necrosis of the femoral head," Surgical Technology International, pp. 247-54, 2016.

[21] M. A. Mont, P. S. Ragland, and G. Etienne, "Core decompression of the femoral head for osteonecrosis using percutaneous multiple small-diameter drilling," Clinical Orthopaedics and Related Research, no. 429, pp. 131-138, 2004.

[22] K. J. Bozic, D. Zurakowski, and T. S. Thornhill, "Survivorship analysis of hips treated with core decompression for nontraumatic osteonecrosis of the femoral head," The Journal of Bone \& Joint Surgery, vol. 81, no. 2, pp. 200-209, 1999.

[23] D. Li, M. Li, P. Liu, Y. Zhang, L. Ma, and F. Xu, "Core decompression or quadratus femoris muscle pedicle bone grafting for nontraumatic osteonecrosis of the femoral head: A randomized 
control study," Indian Journal of Orthopaedics, vol. 50, no. 6, pp. 629-635, 2016.

[24] S. Landgraeber, S. Warwas, T. Claßen, and M. Jäger, "Modifications to advanced Core decompression for treatment of Avascular necrosis of the femoral head," BMC Musculoskeletal Disorders, vol. 18, no. 1, p. 479, 2017.

[25] F. Sadile, A. Bernasconi, F. Carbone, F. Lintz, and G. Mansueto, "Histological fibrosis may predict the failure of core decompression in the treatment of osteonecrosis of the femoral head," International Journal of Surgery, vol. 44, pp. 303-308, 2017.

[26] J. Li, Z. Li, H. Zhang, X. Su, K. Wang, and Y. Yang, "Long-term outcome of multiple small-diameter drilling decompression combined with hip arthroscopy versus drilling alone for early avascular necrosis of the femoral head," Chinese Medical Journal, vol. 130, no. 12, pp. 1435-1440, 2017.

[27] C. J. Wang, F. S. Wang, C. C. Huang, K. D. Yang, L. H. Weng, and H. Y. Huang, "Treatment for osteonecrosis of the femoral head: comparison of extracorporeal shock waves with core decompression and bone-grafting," The Journal of Bone and Joint Surgery American, vol. 87, no. 11, pp. 2380-2387, 2005.

[28] J. Hausdorf, A. Lutz, H. Rohrig, and M. Maier, "Extracorporeal shock wave therapy and femur head necrosis-pressure measurements in the femur head," Zeitschrift für Orthopädie und ihre Grenzgebiete, vol. 142, no. 2, pp. 122-126, 2004.

[29] M. C. Vulpiani, M. Vetrano, D. Trischitta et al., "Extracorporeal shock wave therapy in early osteonecrosis of the femoral head: prospective clinical study with long-term follow-up," Archives of Orthopaedic and Trauma Surgery, vol. 132, no. 4, pp. 499-508, 2012.

[30] J. Y. Lee, J. W. Kwon, J. S. Park, K. Han, W. J. Shin, and J. G. Lee, "Osteonecrosis of femoral head treated with extracorporeal shock wave therapy: analysis of short-term clinical outcomes of treatment with radiologic staging," Hip \& Pelvis, vol. 27, no. 4, pp. 250-257, 2015.

[31] F. Gao, W. Sun, Z. Li, W. Guo, W. Wang, L. Cheng et al., "Highenergy extracorporeal shock wave for early stage osteonecrosis of the femoral head: a single-center case series. Evidencebased complementary and alternative medicine," EvidenceBased Complementary and Alternative Medicine, vol. 2015, Article ID 468090, 2015.

[32] H. Ma, D. Zhou, D. Li, W. Zhang, and B. Zeng, "A histomorphometric study of necrotic femoral head in rabbits treated with extracorporeal shock waves," Journal of Physical Therapy Science, vol. 29, no. 1, pp. 24-28, 2017.

[33] C. d'Agostino, P. Romeo, V. Lavanga, S. Pisani, and V. Sansone, "Effectiveness of extracorporeal shock wave therapy in bone marrow edema syndrome of the hip," Rheumatology International, vol. 34, no. 11, pp. 1513-1518, 2014.

[34] M. Meloni, W. Hoedemaeker, and V. Fornasier, "Failed vascularized fibular graft in treatment of osteonecrosis of the femoral head. A histopathological analysis," Joints, vol. 4, no. 1, pp. 2430, 2017.

[35] S. Yin, C. Zhang, D. Jin, S. Chen, Y. Sun, and J. Sheng, "Treatment of osteonecrosis of the femoral head in lymphoma patients by free vascularised fibular grafting," International Orthopaedics, vol. 35, no. 8, pp. 1125-1130, 2011.

[36] W. C. Eward, C. A. Rineer, J. R. Urbaniak, M. J. Richard, and D. S. Ruch, "The Vascularized Fibular Graft in Precollapse Osteonecrosis: Is Long-term Hip Preservation Possible?” Clinical Orthopaedics and Related Research, vol. 470, no. 10, pp. 2819-2826, 2012.
[37] S. Y. Kim, Y. G. Kim, P. T. Kim, J. C. Ihn, B. C. Cho, and K. H. Koo, "Vascularized compared with nonvascularized fibular grafts for large osteonecrotic lesions of the femoral head," The Journal of Bone and Joint Surgery American, vol. 87, no. 9, pp. 2012-2018, 2005.

[38] D. G. Sotereanos, A. Y. Plakseychuk, and H. E. Rubash, "Free vascularized fibula grafting for the treatment of osteonecrosis of the femoral head," Clinical Orthopaedics and Related Research, no. 344, pp. 243-256, 1997.

[39] A. V. Korompilias, A. E. Beris, M. G. Lykissas, I. P. KostasAgnantis, and P. N. Soucacos, "Femoral head osteonecrosis: why choose free vascularized fibula grafting," Microsurgery, vol. 31, no. 3, pp. 223-228, 2011.

[40] Y. Zhang, L. Li, Z. J. Shi, J. Wang, and Z. H. Li, "Porous tantalum rod implant is an effective and safe choice for early-stage femoral head necrosis: a meta-analysis of clinical trials," European Journal of Orthopaedic Surgery \& Traumatology: Orthopedie Traumatologie, vol. 23, no. 2, pp. 211-217, 2013.

[41] J. Ma, W. Sun, F. Gao, W. Guo, Y. Wang, and Z. Li, "Porous Tantalum Implant in Treating Osteonecrosis of the Femoral Head: Still a Viable Option?" Scientific Reports, vol. 6, no. 1, 2016.

[42] C. J. Veillette, H. Mehdian, E. H. Schemitsch, and M. D. McKee, "Survivorship analysis and radiographic outcome following tantalum rod insertion for osteonecrosis of the femoral head," The Journal of Bone and Joint Surgery American, vol. 88, Suppl 3, pp. 48-55, 2006.

[43] M. Tanzer, J. D. Bobyn, J. J. Krygier, and D. Karabasz, "Histopathologic retrieval analysis of clinically failed porous tantalum osteonecrosis implants," The Journal of Bone \& Joint Surgery, vol. 90, no. 6, pp. 1282-1289, 2008.

[44] M. A. Mont, A. C. Fairbank, K. A. Krackow, and D. S. Hungerford, "Corrective osteotomy for osteonecrosis of the femoral head," The Journal of Bone and Joint Surgery American, vol. 78, no. 7, pp. 1032-1038, 1996.

[45] K. Sonoda, T. Yamamoto, G. Motomura, Y. Nakashima, R. Yamaguchi, and Y. Iwamoto, "Outcome of transtrochanteric rotational osteotomy for posttraumatic osteonecrosis of the femoral head with a mean follow-up of 12.3 years," Archives of Orthopaedic and Trauma Surgery, vol. 135, no. 9, pp. 1257-1263, 2015.

[46] H.-G. Simank, D. R. C. Brocai, C. Brill, and M. Lukoschek, "Comparison of results of core decompression and intertrochanteric osteotomy for nontraumatic osteonecrosis of the femoral head using cox regression and survivorship analysis," The Journal of Arthroplasty, vol. 16, no. 6, pp. 790-794, 2001.

[47] A. A. Sallam, M. A. Imam, K. S. Salama, and O. A. Mohamed, "Inverted femoral head graft versus standard core decompression in nontraumatic hip osteonecrosis at minimum 3 years follow-up," Hip International, vol. 27, no. 1, pp. 74-81, 2017.

[48] M. Noguchi, T. Kawakami, and H. Yamamoto, "Use of vascularized pedicle iliac bone graft in the treatment of avascular necrosis of the femoral head," Archives of Orthopaedic and Trauma Surgery, vol. 121, no. 8, pp. 437-442, 2001. 


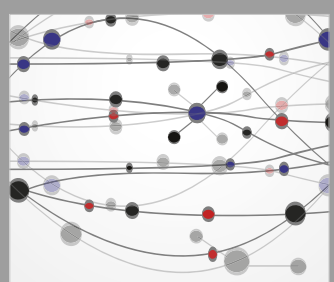

The Scientific World Journal
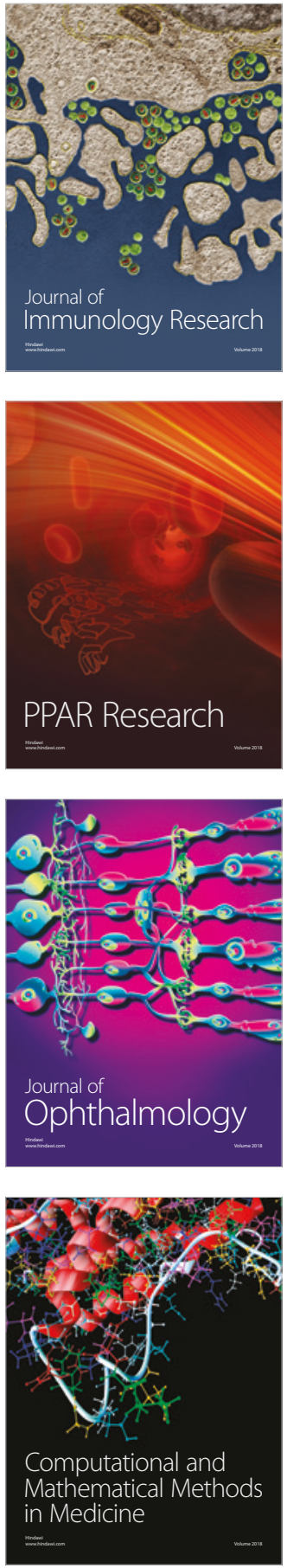

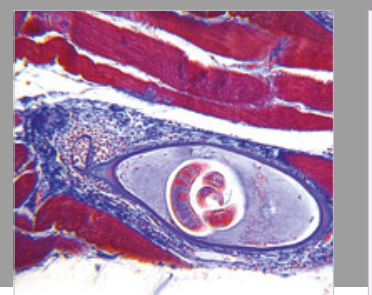

Gastroenterology Research and Practice

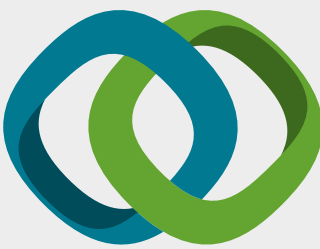

\section{Hindawi}

Submit your manuscripts at

www.hindawi.com
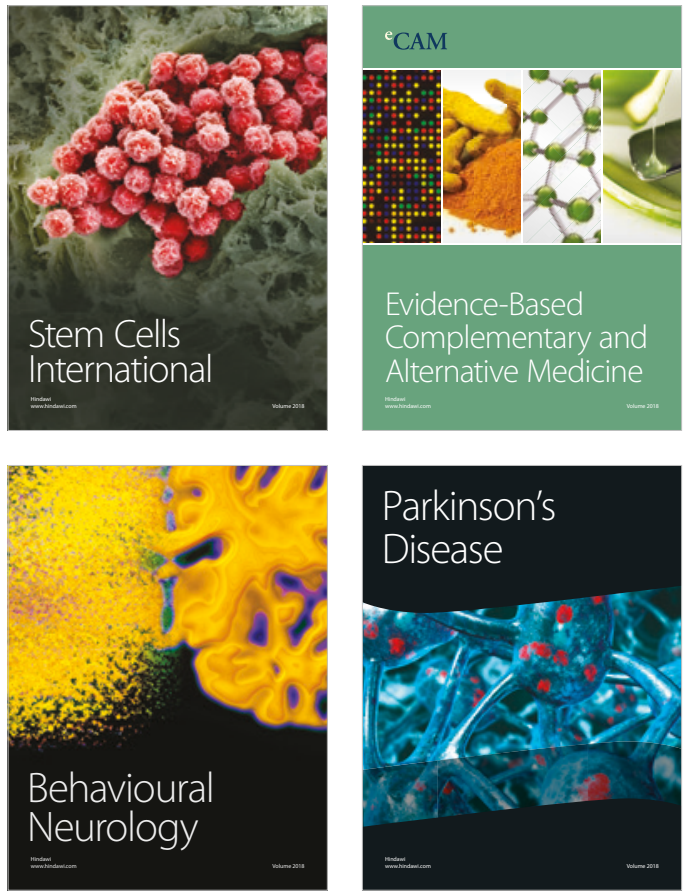

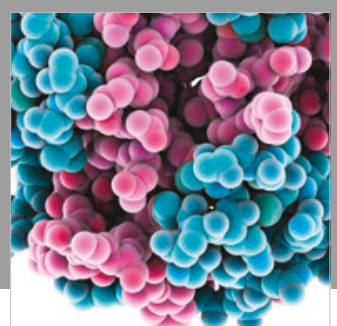

ournal of

Diabetes Research

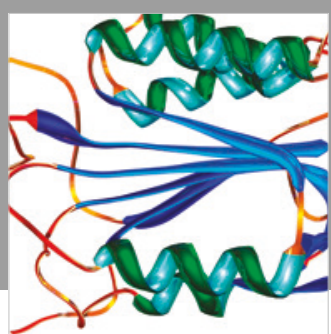

Disease Markers
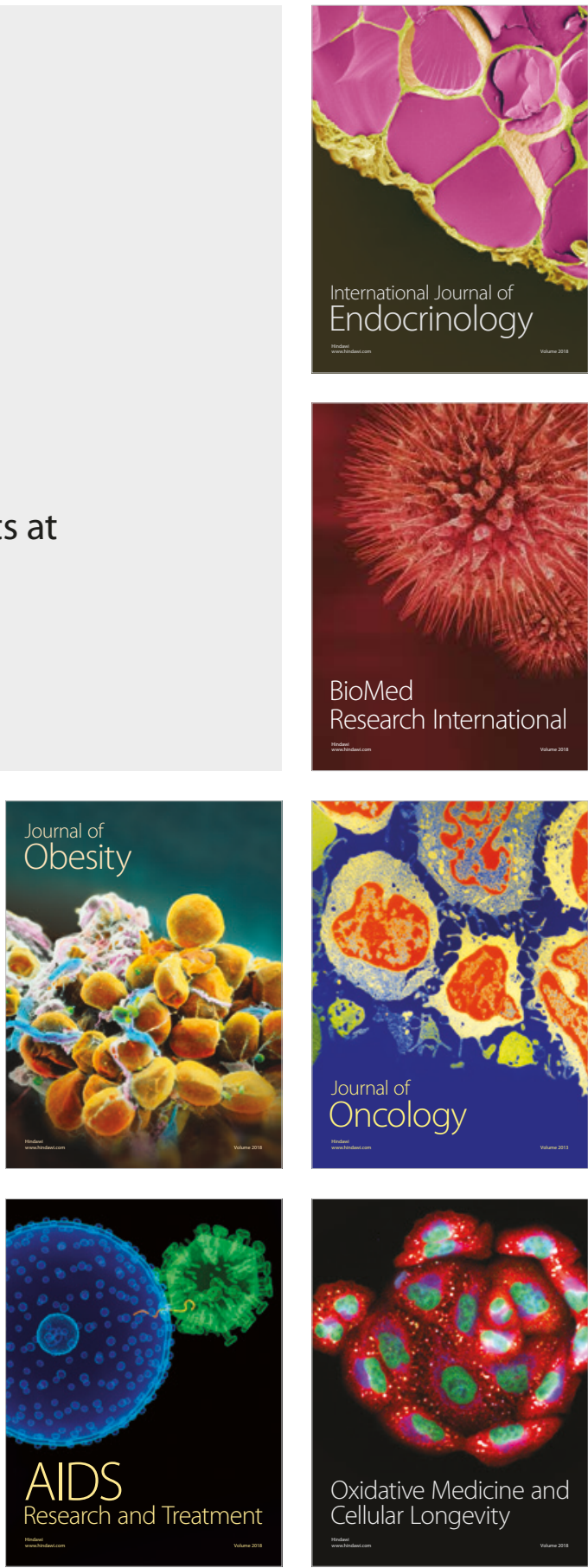